\title{
Necrose tubular aguda e anemia grave após uso intermitente de rifampicina
}

\author{
Acute tubular necrosis and severe anemia \\ after intermittent use of rifampicin
}

\author{
Eduardo Santos ${ }^{1}$, Carla Dias 2 , Paula Dias ${ }^{3}$, Jorge Almeida ${ }^{3}$ \\ ${ }^{1}$ Serviço de Medicina Interna, Centro Hospitalar do Baixo Vouga (CHBV). Aveiro. Portugal \\ ${ }^{2}$ Serviço de Medicina Interna, Centro Hospitalar de S. João (CHSJ). Porto. Portugal \\ ${ }^{3}$ Unidade de Cuidados Intermédios de Medicina, Serviço de Medicina Interna, Centro Hospitalar de S. João (CHSJ). Porto. Portugal
}

\begin{abstract}
Resumo
A rifampicina (RFP) pode ser administrada diariamente ou num esquema intermitente. Quando é administrada de forma intermitente pode produzir reações imuno-alérgicas graves. Apresentamos o caso clínico de um paciente que, após tratamento com RFP de forma intermitente para uma infeção por Mycobacterium intracellulare, desenvolveu quadro de necrose tubular aguda, anemia, prolongamento dos tempos de coagulação e citólise hepática ligeira. Após suspensão do fármaco houve recuperação em 24h das alterações da coagulação e da citólise hepática, tendo tido no entanto necessidade de hemodiálise por persistência de anúria e uremia, e transfusão de concentrados de eritrócitos pela gravidade da anemia. Teve alta com recuperação de todas as disfunções.
\end{abstract}

Palabras clave: Rifampicina. Insuficiencia renal aguda. Hepatitis. Coagulación intravascular diseminad. Anemia hemolítica.

\section{Introdução}

A rifampicina (RFP) é um antibiótico semissintético derivado das rifamicinas, um grupo de agentes antibacterianos produzidos pelo fungo Amycolatosis mediterranei. A RFP foi inicialmente desenvolvida para tratar a tuberculose, mas, mais recentemente, tem sido usada contra outras bactérias ${ }^{1}$, podendo ser administrada diariamente ou num esquema intermitente ${ }^{2,3}$.

Quando a RFP é administrada de forma diária, os possíveis efeitos adversos incluem reações alérgicas mediadas por IgE, erupções cutâneas, desconforto gastrointestinal, hepatotoxicidade, e interações medicamentosas, mas quando administrada de forma intermitente pode produzir efeitos adversos mais graves conhecidos como reações imunomediadas por lgG e IgM, dirigidas contra os eritrócitos, plaquetas e outras células alvo que expressam 0 antigénio "I" do sangue, incluindo as células epiteliais tubulares renais².

A administração RFP, mesmo em doses pouco usuais, de forma intermitente ou diária, pode resultar na sensibilização com um aumento rápido no título de anticorpos após a exposição repetida ao fármaco². 0 tratamento com uma única dose de RFP pode induzir sensibilização, resultando em reações imuno-alérgicas após reexposição, enquanto a administração diária de RFP poderá conferir tolerância imunológica contra estas reações².

\begin{abstract}
Rifampicin (RFP) may be administered on a daily or intermittent base. When administered intermittently it can produce severe immunoallergic reactions. We present a report of a patient who developed acute tubular necrosis, anemia, prolongation of clotting times and mild hepatic cytolysis after therapy with RFP on an intermittent basis directed to a Mycobacterium intracellulare infection. After suspension of the drug the patient recovered from clotting disturbance and hepatic cylolysis in $24 \mathrm{~h}$, but required hemodialysis for persistent anuria and uremia, and packed cells transfusion because of the severity of anemia. He was discharged with total recovery of all those dysfunctions.
\end{abstract}

Keywords: Rifampicin. Acute renal failure. Disseminated intravascular coagulation. Hepatitis. Hemolytic anemia.

Apresentamos um paciente que teve uma reação imunoalérgica grave, após tratamento com RFP para uma infeção por Mycobacterium intracellulare.

\section{Caso clínico}

Doente do sexo masculino, de 69 anos de idade, admitido na nossa Unidade por quadro de insuficiência renal aguda (IRA) oligúrica, anemia e elevação dos enzimas de citólise hepática. Tratava-se de ex-fumador de 60 unidades-maço/ano, com doença pulmonar obstrutiva crónica e sequelas de tuberculose pulmonar (TP) tratada em 2004, durante 9 meses.

Em Agosto de 2011 iniciou quadro de tosse produtiva e astenia. Imagiologicamente na radiografia torácica apresentava infiltrado bilateral, além de alterações sequelares de TP (fibrose e bronquiectasias de tração). A pesquisa de bacilos ácido-álcool resistentes (BAAR) na expectoração foi positiva, pelo que iniciou fármacos antituberculosos (rifampicina $600 \mathrm{mg} / \mathrm{dia}$, isoniazida 300 mg/dia, pirazinamida 2000 mg/dia e etambutol $1600 \mathrm{mg} / \mathrm{dia}$ ) por suspeita de reactivação de TP, após o que teve quadro de IRA com necessidade de hemodiálise, tendo recuperado a função renal. Nessa altura suspendeu todos os fármacos antituberculosos.

Em Setembro de 2011 foi identificado Mycobacterium intracellulare em 3 amostras de expetoração colhidas em Agosto de 2011 e, uma vez que reunia todos os critérios para diagnóstico de doença pulmonar por micobactérias não tuberculosas (tabela 1), com indicação para tratamento ${ }^{4}$, iniciou etambutol $1600 \mathrm{mg} / \mathrm{dia}$ e claritromicina $500 \mathrm{mg}$ bid em ambulatório, tendo sido internado no Serviço de Pneumologia em Outubro de 2011 para início de 
tratamento com rifampicina, em doses crescentes até $600 \mathrm{mg} /$ dia, sob vigilância. À $6^{a}$ toma de rifampicina sofreu agravamento dos valores séricos de ureia (63 mg/dl), aspartato aminotransferase (AST) - $51 \mathrm{u} / \mathrm{l}$ - e gama-glutamiltranspeptidase (GGT) - 100 u/l -, motivo pelo qual suspendeu a sua toma durante 6 dias, até normalização destes valores. Após este período, retomou a RFP, agora apenas com $300 \mathrm{mg} / \mathrm{dia}$ e, 18h depois, iniciou quadro de IRA oligúrica (ureia $138 \mathrm{mg} / \mathrm{dl}$ e creatinina 5,6 mg/dl), anemia ( $\mathrm{Hb}$ 8,6 g/dl) e elevação da AST (81 u/l) tendo sido admitido na nossa Unidade para diagnóstico e tratamento.

Tabela 1. Criterios de diagnostico de doença pulmonar por micobacterias não tuberculosas em doentes seronegativos para VIH e sem outro factor de imunodepressão grave

\section{Critérios clínicos}

A) Sinais ou sintomas compatíveis (tosse, fadiga, febre, perda de peso, hemoptises, dispneia) com deterioração do estado clínico e B) Exclusão de outras doenças que expliquem o quadro

\section{Critérios imagiológicos}

A) Qualquer uma das seguintes alterações na radiografia do tórax

- Infiltrados, com ou sem nódulos (persistentes desde há > 2 meses ou com agravamento progressivo);

Cavitação;

- Nódulos ou

B) Qualquer uma das seguintes alterações na tomografia

computorizada de alta resolução

- Nódulos pequenos múltiplos;

Bronquiectasias multifocais, com ou sem nódulos pulmonares.

\section{Critérios bacteriológicos}

A) Pelo menos 3 amostras de expetoração ou lavado broncoalveolar

(LBA) no intervalo de 1 ano com

- 3 culturas positivas, com pesquisas de BAAR negativas ou

2 culturas positivas e 1 pesquisas de BAAR positiva ou

B) Apenas disponível um LBA

- Cultura positiva com crescimento de $2+, 3+$ ou $4+$ ou

- Cultura positiva com pesquisa de BAAR de 2+, 3+ ou 4+ ou

C) Biopsia

- Crescimento positivo na cultura de tecido pulmonar ou

- Granuloma e / ou pesquisa de BAAR positiva no tecido pulmonar, com $>1$ culturas de expetoração / LBA positivas ou

- Crescimento positivo na cultura de tecido extrapulmonar

Nota: Para se fazer o diagnóstico de doença pulmonar por micobactérias não tuberculosas, têm que ser cumpridos todos os critérios - clínicos, imagiológicos e bacteriológicos.

Na nossa Unidade realizou estudo complementar que foi sugestivo de necrose tubular aguda (fração de excreção de sódio - 2,8\%; rácio azoto ureico:creatinina - 11,8:1; sem eosinofilia ou eosinofilúria), não tendo sido realizada biopsia renal, pela evolução do doente. A serologia para o Vírus da Imunodeficiência Humana (VIH) foi negativa. 0 doente apresentava ainda distúrbio da coagulação, em relação com provável coagulação intravascular disseminada (CID), com prolongamento dos tempos de coagulação e elevação dos $\mathrm{D}$ dímeros (tabela 2), embora sem fenómenos trombóticos, hemorrágicos ou trombocitopenia. 0 estudo etiológico da anemia foi sugestivo de anemia hemolítica não imune, com teste de Coombs direto negativo, hiperbilirrubinemia (total $-2,73 \mathrm{mg} / \mathrm{dl}$, indirecta - 1,59 mg/dl) e lactato desidrogenase (LDH) - $1528 \mathrm{u} / \mathrm{l}$, embora com pouco consumo de haptoglobina e menos de $2 \%$ de esquizócitos no sangue periférico.
Foi admitida toxicidade pela rifampicina como causa do quadro, pelo que se suspendeu o fármaco e se iniciou fluidoterapia. As alterações da coagulação e da citólise hepática reverteram $24 \mathrm{~h}$ depois, apenas com a suspensão da RFP, sem necessidade de administração de plasma. Ao $3^{\circ}$ dia, por persistência da oligúria e uremia, iniciou hemodiálise, tendo recuperado diurese após a $3^{\mathrm{a}}$ sessão. Pela gravidade da anemia, teve necessidade de transfusão de 4 unidades de concentrado de eritrócitos.

Foi transferido para o Serviço de Pneumologia após 5 dias de internamento na nossa Unidade, tendo tido alta 17 dias depois, já sem anemia ou disfunção renal.

Tabela 2. Resultados laboratoriais

\begin{tabular}{|c|c|c|c|c|c|c|c|}
\hline & D-0 & D-1 & D-2 & D-3 & D-4 & D-5 & D-6 \\
\hline Hemoglobina (g/dl) & 10,6 & 8,6 & 7,0 & 8,8 & 8,6 & 7,9 & 9,6 \\
\hline Leucócitos (G/l) & 11,17 & 46,45 & 27,85 & 24,19 & 15,35 & 13,31 & 10,81 \\
\hline Neutrófilos (\%) & 63,5 & 92,3 & 86,9 & 87,1 & 83,6 & 65,3 & 60,1 \\
\hline Eosinófilos (\%) & 4,2 & 0,2 & 1,7 & 0,0 & 0,1 & 3,8 & 5,0 \\
\hline Linfócitos (\%) & 22,0 & 2,6 & 6,0 & 6,2 & 7,3 & 15,8 & 17,4 \\
\hline Plaquetas (G/l) & 447 & 278 & 221 & 213 & 186 & 163 & 148 \\
\hline Fibrinogénio (mg/dl) & & 264 & & 416 & & & \\
\hline$D$ dímeros $(\mu \mathrm{g} / \mathrm{ml})$ & & 46,75 & & 6,28 & & & \\
\hline TP (seg.) & & 21,9 & 13,0 & & & & \\
\hline APTT (seg.) & & 46,2 & 29,1 & & & & \\
\hline Ureia (mg/dl) & 23 & 138 & 149 & 176 & 150 & 115 & 58 \\
\hline Creatinina (mg/dl) & 0,9 & 5,6 & 6,6 & 7,5 & 6,9 & 5,7 & 4,0 \\
\hline AST $(\mathrm{u} / \mathrm{l})$ & 25 & 81 & 45 & 31 & 35 & & 30 \\
\hline $\mathrm{ALT}(\mathrm{u} / \mathrm{l})$ & 34 & 38 & 33 & 29 & 43 & & 40 \\
\hline $\mathrm{LDH}(\mathrm{u} / \mathrm{l})$ & & 1528 & 1010 & 758 & 616 & 489 & 439 \\
\hline GGT (u/l) & & 90 & 68 & 83 & & & \\
\hline $\mathrm{FA}(\mathrm{u} / \mathrm{l})$ & & 87 & 88 & 79 & & & \\
\hline $\mathrm{BT}(\mathrm{mg} / \mathrm{dl})$ & 0,56 & 2,73 & 1,66 & 1,23 & 1,08 & & 1,16 \\
\hline $\mathrm{BC}(\mathrm{mg} / \mathrm{dl})$ & 0,17 & 1,14 & 0,7 & 0,5 & 0,42 & & 0,37 \\
\hline
\end{tabular}

TP - tempo de protrombina; APTT - tempo de tromboplastina parcial ativada; AST - aspartato aminotransferase; ALT - alanina aminotransferase; LDH - lactato desidrogenase; GGT - gamaglutamiltranspeptidase; FA - fosfatase alcalina; BT - bilirrubina total; BC - bilirrubina conjugada;

\section{Discussão}

0 tratamento actualmente recomendado para as infecções pulmonares por Micobacterium avium complex (MAC) é claritromicina ( $500 \mathrm{mg}$ bid) ou azitromicina ( $250 \mathrm{mg} /$ dia ou 500 $\mathrm{mg} 3$ vezes por semana), rifampicina (600 mg/dia) ou rifabutina (300 mg/dia), e etambutol ( $25 \mathrm{mg} / \mathrm{kg} / \mathrm{dia} 2$ meses, e depois $15 \mathrm{mg} / \mathrm{kg} / \mathrm{dia}$ ). Se 0 doente tolerar, poderá associarse ainda estreptomicina $(500 \mathrm{mg}-1000 \mathrm{mg} 3$ vezes por semana), durante as primeiras 8-12 semanas. Este tratamento deverá sem mantido até as culturas serem negativas durante $1 \mathrm{ano}^{4}$. Em caso de toxicidade à rifampicina, com necessidade da sua suspensão, o fármaco de eleição deverá ser uma fluoroquinolona (levofloxacina 750 - $1000 \mathrm{mg} / \mathrm{dia}$ ou ofloxacina $400 \mathrm{mg}$ bid), pela sua actividade bactericida e boa distribuição tecidular, devendo manter-se a terapêutica de associação, pela elevação ocorrência de resistências entre as fluoroquinolonas 5 . 
Na literatura encontramos vários relatos isolados de casos de IRA ${ }^{6-8}$, hepatite ${ }^{9-11}, \mathrm{CID}^{2,12,13}$ e anemia hemolítica ${ }^{14,15}$, após a administração intermitente de RFP. 0 nosso paciente destaca-se pela gravidade do quadro clínico, tendo apresentado simultaneamente IRA, anemia e, ainda que com menor gravidade, prolongamento dos tempos de coagulação e citólise hepática. A gravidade do nosso doente provavelmente não terá sido maior pelo diagnóstico precoce da situação de toxicidade e atempada suspensão da RFP.

A IRA associada à toma de RFP geralmente ocorre em pacientes que tomam o fármaco de forma intermitente, começando tipicamente após reexposição ao fármaco, com apenas alguns casos isolados relatados no regime diário6,8. A RFP causa IRA devido à formação de imuno-complexos com o fármaco e posterior ligação destes ao antigénio "I" das células tubulares renais, levando a dano tubular e intersticial no rim ${ }^{6,7}$. Os principais padrões histológicos de lesão renal associada ao uso de RFP são a necrose tubular aguda (NTA) e a nefrite intersticial (NI). A NTA é o mais comum, ocorrendo como uma reação fulminante, com sintomas abdominais, hemólise e trombocitopenia. A NI tem sido descrita como insidiosa, com sintomas semelhantes à síndrome gripal. 0 prognóstico da IRA secundária à RFP é geralmente bom ${ }^{6}$. Por norma, a mortalidade é baixa, ocorrendo recuperação completa da função renal. 0 principal fator de prognóstico é a duração da fase de anúria que, se prolongada, se associa a necessidade de diálise e a uma taxa de redução de azotemia lenta ${ }^{12}$.

A hepatite é uma reação invulgar à toma de RFP, ocorrendo em menos de $1 \%$ dos pacientes ${ }^{9}$, sendo mais frequente $(2,6 \%)$ com a coadministração de Isoniazida ${ }^{10}$. 0 mecanismo de patogénese da hepatotoxicidade da RFP não está totalmente esclarecido. Embora possa existir uma toxicidade dose-dependente, não foi ainda demonstrada uma correlação direta entre os níveis séricos e a hepatotoxicidade. A hipersensibilidade pode ser uma possibilidade. Uma alteração nos níveis de antioxidantes, com aumento da peroxidação lipídica, pode sugerir um mecanismo oxidativo para a hepatotoxicidade induzida pela RFP9 . A desnutrição pode ser um fator de risco, levando à depleção das reservas de glutatião e fazendo com que os pacientes fiquem mais vulneráveis a lesões oxidativas ${ }^{11}$. Os pacientes com doença hepática pré-existente correm maior risco, tendo sido observado um aumento do risco de hepatotoxicidade em pacientes com aminotransferase basais anormais ${ }^{10,11}$. Os pacientes geralmente recuperam completamente após suspensão da RFP9 .

A incidência de CID após uso intermitente de RFP é baixa, mas pode ocorrer repentinamente após reexposição, em alguns casos logo nos primeiros 30 minutos após a ingestão. Acredita-se que a CID resulte da ativação do complemento mediada por anticorpos². A RFP pode funcionar como um hapteno, levando à formação de anticorpos anti-RFP12,14. Após a reexposição ao fármaco, ocorre formação de com- plexos RFP-anticorpo que, ou se ligam ao complemento no sangue, ou são primeiro absorvidos pela membrana dos glóbulos vermelhos e plaquetas, ativando de seguida 0 complemento e levando à destruição das células, provocando anemia e trombocitopenia imunes ${ }^{2,12}$. A exposição aos constituintes dos eritrócitos destruídos, leva à ativação da cascata de coagulação ${ }^{2}$. A abordagem da CID induzida pela RFP consiste na suspensão do fármaco e transfusão de plaquetas, concentrados de eritrócitos e plasma ${ }^{2,12}$.

A hemólise aguda raramente surge como reação adversa à RFP, podendo ser encontrada apenas nos pacientes que tomam o fármaco de forma intermitente ou irregular ${ }^{14}$. 0 mecanismo de hemólise associada à RFP é similar ao da CID, resultando da ativação do complemento mediada por imuno-complexos RFP-anticorpo ${ }^{12,14}$. Apesar de rara, a hemólise secundária à RFP pode provocar anemia grave, devendo os clínicos estar atentos para a possibilidade da sua ocorrência. 0 tratamento consiste em evicção do fármaco e transfusão de concentrado de eritrócitos ${ }^{14}$.

Os clínicos devem estar alerta para este tipo de manifestação de reações imuno-alérgicas à RFP, que podem ocorrer durante 0 tratamento intermitente ou irregular. É fundamental a colheita de uma história farmacológica detalhada para se fazer esse diagnóstico. Em doentes com reações imunoalérgicas à RFP, esta deve ser interrompida permanentemente, dada a gravidade dessas reações, podendo mesmo ser fatais². A administração diária parece ser mais segura ${ }^{2,3}$.

\section{Bibliografía}

1. Prince MI, Burt AD, Jones DEJ: Hepatitis and liver dysfunction with RFP therapy for pruritus in primary biliary cirrhosis. Gut 2002;50:436-439

2. Havey TC, Cserti-Gazdewich C, Sholzberg M, Keystone JS, Gold WL: Recurrent Disseminated Intravascular Coagulation Caused by Intermittent Dosing of Rifampin. Am J Trop Med Hyg 2012; 86(2):264-267

3. Aquinas M, Allan WGL, Horsfall PAL,et al: Adverse Reactions to Daily and Intermittent RFP Regimens for Pulmonary Tuberculosis in Hong Kong. Brit Med J 1972; 1:765-771

4. American Thoracic Society: Diagnosis and Treatment of Disease Caused by Nontuberculous Mycobacteria. Am J Respir Crit Care Med 1997; 156:S1-S25

5. Schubert C, Bates WD, Moosa MR: Acute tubulointerstitial nephritis related to antituberculous drug therapy. Clinical Nephrology 2010; 73 (6):413-419

6. van der Meulen J, de Jong GMT, Westenend PJ: Acute interstitial nephritis during RFP therapy can be a paradoxical response: a case report. Cases Journal 2009; 2:6643

7. Flynn CT, Rainford DJ, Hope E: Acute Renal Failure and RFP: Danger of Unsuspected Intermittent Dosage. Brit Med J 1974; 2:482

8. Girling DJ, Hitze KL: Adverse reactions to RFP. Bulletin of the World Health Organization 1979; 57(1):45-49

9. Yew WW, Leung CC: Antituberculosis drugs and hepatotoxicity. Respirology 2006; 11:699-707

10. Makhlouf HA, Helmy A, Fawzy E, El-Attar M, Rashed HAG: A prospective study of antituberculous drug-induced hepatotoxicity in an area endemic for liver diseases. Hepatol Int 2008; 2:353-360

11. Prince MI, Burt AD, Jones DEJ: Hepatitis and liver dysfunction with RFP therapy for pruritus in primary biliary cirrhosis. Gut 2002; 50:436-439

12. Costiniuk CT, McCarthy AE, Talreja $\mathrm{H}$, et al: Acute renal failure and disseminated intravascular coagulation associated with rifampin in tuberculosis treatment. Int $\mathrm{J}$ Tuberc Lung Dis 2011; 15(3):421-424

13. Fujita M, Kunitake R, Nagano Y, Maeda F: Disseminated Intravascular Coagulation Associated with Pulmonary Tuberculosis. Internal Medicine 1997; 36: 218-220

14. Oguz A, Kanra T, Gokalp A, Gultekin A: Acute Hemolytic Anemia Caused By Irregular Rifampicin Therapy. Turk J Pediatr 1989; 31:83-88

15. Ahrens N, Genth R, Salama A: Belated diagnosis in three patients with rifampicininduced immune haemolytic anaemia. Br J Haematol 2002; 117: 441-443 